\title{
Apatinib in the treatment of advanced lung adenocarcinoma with KRAS mutation
}

This article was published in the following Dove Press journal:

OncoTargets and Therapy

28 August 2017

Number of times this article has been viewed

\section{Da-xiong Zeng* \\ Chang-guo Wang* \\ Jian-an Huang \\ Jun-hong Jiang}

Department of Respiratory and Critical Care, The First Affiliated Hospital of Soochow University, Suzhou, Jiangsu, People's

Republic of China

*These authors contributed equally to this work
Correspondence: Jun-hong Jiang Department of Respiratory and Critical Care, The First Affiliated Hospital of Soochow University, Suzhou, Jiangsu 215006, People's Republic of China Tel +8651267972092 Email jiang20001969@163.com

\begin{abstract}
Activating KRAS mutations in lung adenocarcinoma are characterized with treatment resistance and poor prognosis. As a small molecule inhibitor of vascular endothelial growth factor receptor-2 (VEGFR-2) tyrosine kinase, apatinib has been proven successful in advanced gastric cancer and breast cancer. In this study, we show the result of apatinib as salvage treatment in lung adenocarcinoma patients with KRAS mutation. Four advanced lung adenocarcinoma patients with KRAS mutation were orally administered apatinib $(250 \mathrm{mg} / \mathrm{d})$ after second-line treatment. One patient showed progressive disease, while 3 patients showed stable disease response to apatinib, with a median progression-free survival (PFS) of 3.8 months (1.5-5.5 months). The main toxicities were hoarseness and hemoptysis, which were manageable. Therefore, apatinib might be an optional choice for advanced lung adenocarcinoma patients with KRAS mutation in post second-line treatment.
\end{abstract}

Keywords: lung cancer, apatinib, VEGFR-2, KRAS mutation

\section{Introduction}

Lung cancer is the leading cause of cancer-related death worldwide. Non-small-cell lung cancer (NSCLC) accounts for $80 \%$ of lung cancer patients and is associated with multiple different oncogenic driver mutations. ${ }^{1}$ Activating KRAS genetic driver mutation, noted in $15 \%-25 \%$ of NSCLC patients, is an important frequent recurring molecular event in lung adenocarcinoma. ${ }^{2}$ However, NSCLC with KRAS mutation is associated with aggressive biology and is refractory to current clinical therapies. Little progress has been made in the treatment of KRAS-mutated NSCLC during the past decades.

Angiogenesis is an important feature in tumor growth, development and metastasis. ${ }^{3}$ Vascular endothelial growth factor (VEGF) signaling promotes angiogenesis via activation of VEGF receptor (VEGFR), which is a critical target of cancer treatment. ${ }^{4}$ Agents targeting VEGF or VEGFR have been implicated in several kinds of solid tumors. Bevacizumab, the antibody against VEGF, significantly improved prognosis in advanced nonsquamous NSCLC when added to chemotherapy. ${ }^{5}$ Another VEGFR antibody, ramucirumab, also showed encouraging efficacy in advanced NSCLC treatment. ${ }^{6}$ However, these agents were both suggested as combinations with platinumbased doublet chemotherapy in first-line treatment. There was little evidence for antiangiogenesis drug monotherapy as post second-line therapy in advanced lung adenocarcinoma, especially in KRAS-mutated patients.

Apatinib is an oral small molecule inhibitor of VEGFR-2 tyrosine kinase. Previous reports have demonstrated its efficiency in advanced breast and gastric cancer therapy. 7,8 Three recent studies also showed the efficiency of higher dosage (500-825 mg/d) 
apatinib in NSCLC patients. ${ }^{9-11}$ However, the efficiency of apatinib in KRAS-mutated lung adenocarcinoma remains unclear. In this study, we show the results of apatinib administration at a lower dosage $(250 \mathrm{mg} / \mathrm{d})$ in 4 advanced lung adenocarcinoma patients with KRAS mutation.

\section{Patients and methods}

This study was approved by the research ethics committee of the First Affiliated Hospital of Soochow University (ChiCTR-OPN-16009458), and all patients provided written informed consent to have the case details published. Thus, 4 KRAS-mutated advanced lung adenocarcinoma patients were enrolled in this study. The main inclusion criteria included hemoptysis, thrombotic diseases, uncontrolled hypertension and liver or renal insufficiency. The patients were all diagnosed with pathologically advanced lung adenocarcinoma (IV stage), and the next-generation sequences in different periods showed KRAS mutation, with wild-type epidermal growth factor receptor (EGFR) and anaplastic lymphoma kinase (ALK)-negative status. All patients underwent platinum-based doublet chemotherapy as the first-line treatment. Oral apatinib (250 mg/d, Jiangsu Hengrui Medicine Co, Ltd; 28 days for a cycle) was used as salvage treatment for third-line to fourth-line treatment.

Tumor responses were evaluated every 1 month or were assessed when significant signs of progression appeared, according to the Response Evaluation Criteria in Solid Tumors (RECIST 1.1) using chest imaging (computed tomography [CT] scan). Objective tumor responses included complete response (CR), partial response (PR), stable disease (SD) and progressive disease (PD). All the adverse effects were also recorded.

\section{Results}

The characteristics of all patients are shown in Table 1. All patients were male, aged from 56 to 81 years. All patients had Stage IV adenocarcinoma with KRAS mutation. Before oral apatinib treatment $(250 \mathrm{mg} / \mathrm{d})$, all patients underwent first-line and second-line chemotherapy treatments.

One month after apatinib monotherapy, 3 patients showed SD and 1 patient showed PD (Figure 1). The progression-free survival (PFS) periods of the 3 patients were 1.5 months, 4.5 months and 5.5 months, respectively. Only 1 patient showed manageable hoarseness and hemoptysis (Grade 1).

\section{Discussion}

KRAS mutation accounts for 15\%-25\% NSCLC patients. However, recent approaches to inhibit KRAS directly showed little effect in vivo. ${ }^{2}$ For KRAS-mutated patients, chemotherapy with or without antiangiogenic agents is the standard first-line treatment regime. Antibody against VEGF or VEGFR showed benefit in terms of PFS and overall survival (OS) in advanced nonsquamous NSCLC when added to chemotherapy. ${ }^{5,6}$ In this study, all patients received standard platinum-based doublet chemotherapy as first-line treatment. Because of the patients' poor economic status, we did not select bevacizumab or ramucirumab for addition to chemotherapy for first-line treatment. However, there were few choices for subsequent therapy in these patients after disease progression.

In advanced nonsquamous NSCLC, antiangiogenic agent was suggested in combination with chemotherapy. There was little evidence for antiangiogenic agent monotherapy in lung adenocarcinoma patients, except for persistent treatment. Apatinib is a small molecule oral drug for antiangiogenesis treatment, which has been implicated in large clinical trials of metastatic breast or gastric cancer. ${ }^{7,8}$ Three recent reports have focused on apatinib in NSCLC treatment, including adenocarcinoma and squamous carcinoma patients. ${ }^{9-11}$ Their results demonstrated the efficiency of apatinib in NSCLC, including EGFR-mutated patients and EGFR wild-type patients. In this study, we show the

Table I Characteristics of 4 advanced lung adenocarcinoma patients administered apatinib therapy

\begin{tabular}{|c|c|c|c|c|}
\hline Characteristics & Patient I & Patient 2 & Patient 3 & Patient 4 \\
\hline Age, years & 56 & 81 & 73 & 62 \\
\hline Sex & Male & Male & Male & Male \\
\hline Stage & IV $\left(\mathrm{T}_{2} \mathrm{~N}_{2} \mathrm{M}_{\mathrm{Ib}}\right)$ & IV $\left(\mathrm{T}_{2} \mathrm{~N}_{2} \mathrm{M}_{1 \mathrm{~b}}\right)$ & IV $\left(\mathrm{T}_{4} \mathrm{~N}_{3} \mathrm{M}_{\mathrm{Ib}}\right)$ & IV $\left(\mathrm{T}_{3} \mathrm{~N}_{2} \mathrm{M}_{\mathrm{Ib}}\right)$ \\
\hline \multicolumn{5}{|c|}{ Treatment (response) } \\
\hline First-line & Plat + Pem (SD) & Pem (SD) & Carb + Pem (SD) & Carb + Pem (SD) \\
\hline Second-line & Docetaxel (SD) & Docetaxel (PD) & Docetaxel (SD) & Docetaxel (PD) \\
\hline Third-line & Apatinib (SD) & Apatinib (SD) & Gefitinib (PD) & Apatinib (PD) \\
\hline Fourth-line & - & - & Apatinib (SD) & - \\
\hline Apatinib PFS & 4.5 months & 1.5 months & 5.5 months & - \\
\hline Adverse effects & - & Hemoptysis (Grade I) & - & - \\
\hline
\end{tabular}

Abbreviations: Carb, carboplatin; Plat, platinum; PD, progressive disease; Pem, pemetrexed; PFS, progression-free survival; PR, partial response; SD, stable disease. 

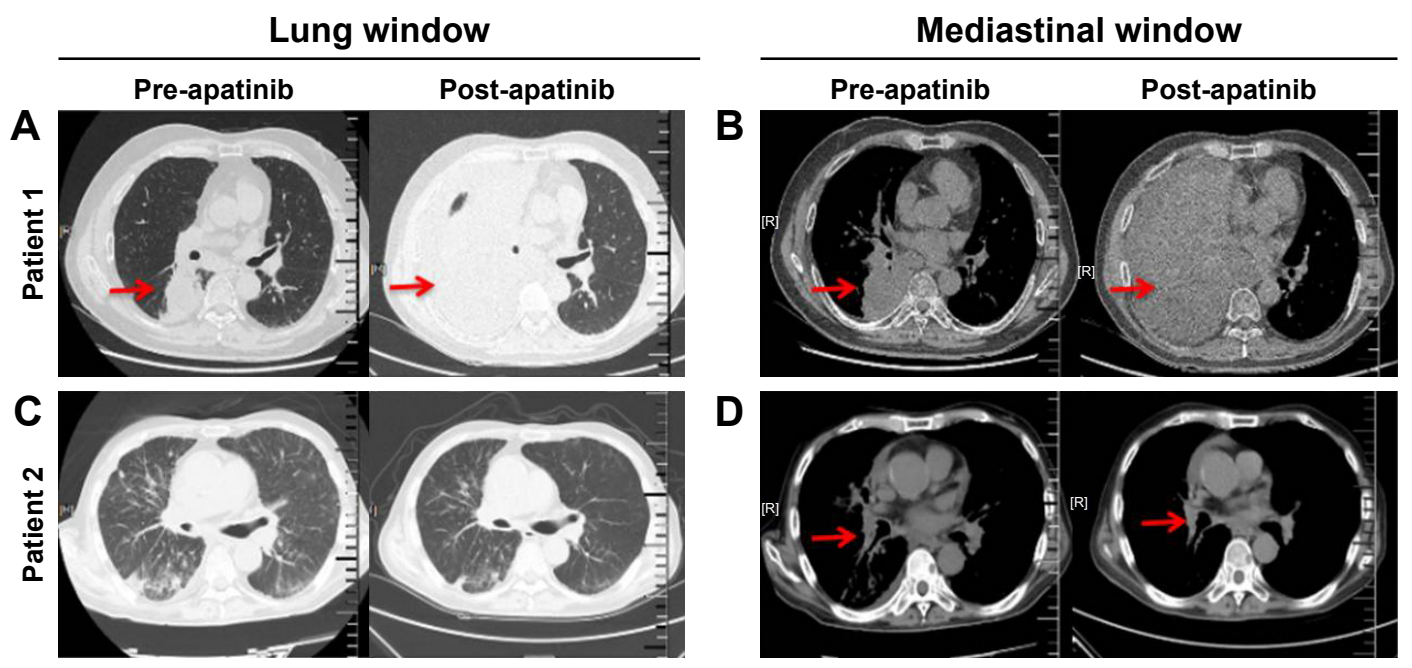

Figure I Representative CT images (lung windows and mediastinal windows) of two patients before and after apatinib monotherapy.

Notes: CT images of Patient I showed progressive disease response. CT images of Patient 2 showed stable disease response. Pre- and post-apatinib images at the (A, C) lung and (B, D) mediastinal windows for Patients I and 2, respectively. The red arrows indicate the tumor variations before and after apatinib therapy.

Abbreviation: $\mathrm{CT}$, computed tomography.

efficiency of apatinib monotherapy in KRAS-mutated lung adenocarcinoma patients for third-line to fourth-line treatment. The median PFS in previous reports was $\sim 4.2$ months. In our study, the median PFS was 3.8 months. These results were similar. These might provided an optional choice for salvage therapy in KRAS-mutated patients. However, there remains a lack of large-scale random controlled clinical trials on apatinib in NSCLC, especially in KRASmutated NSCLC.

Additionally, there were several different points in our report. First, we investigated KRAS-mutated adenocarcinoma patients. This was significantly different from previous studies in adenocarcinoma with or without EGFR mutation. KRAS-mutated NSCLC showed aggressive biology and chemotherapy resistance, frequently resulting in obviously worse prognosis than for EGFR-mutated or wild-type NSCLC patients. Our report might provide some information for a potential choice in KRAS-mutated patients. Second, the dosage of apatinib in our study was $250 \mathrm{mg} / \mathrm{d}$, which was significantly lower than that in a previous report $(500-850 \mathrm{mg} / \mathrm{d})$. It was interesting that a lower dosage brought similar PFS in our study when compared with a higher dosage in previous studies. Moreover, the lower dosage of apatinib showed fewer adverse effects. Only one patient emerged with manageable hoarseness and hemoptysis (Grade 1). Although these results indicated that lower dosage might be an optional choice, the most suitable dosage of apatinib in advanced NSCLC remains unclear. All these problems should be explicitly addressed in further large-scale clinical trials.
In gastric cancer patients, high dose of apatinib $(850 \mathrm{mg} / \mathrm{d})$ was frequently associated with high rate of toxicity, such as hypertension (40.43\%), leukopenia (48.94\%) and hand-foot syndrome $(25.53 \%){ }^{7}$ In a recent report, apatinib $(500 \mathrm{mg} / \mathrm{d})$ also showed a total grade $3 / 4$ toxicity of $50 \% .{ }^{9}$ In our study, a lower dosage of apatinib $(250 \mathrm{mg} / \mathrm{d})$ only resulted in hoarseness and hemoptysis (Grade 1) in a patient without weakening the curative effect. These results indicated that the dosage of apatinib in different cancers might differ significantly.

\section{Conclusion}

This report revealed that apatinib is an optional choice as salvage treatment in advanced KRAS-mutated adenocarcinoma patients. However, further investigations are needed to disclose the solutions to some problems, such as the most suitable dosage, combination with chemotherapy and the efficiency in other rare genetic driver mutations.

\section{Acknowledgments}

This study was supported by the Jiangsu Province Special Program of Medical Science (number BE2016672), the Clinical Key Specialty Project of China, the Clinical Medicine Center of Suzhou (number SZZX201502), Suzhou Key Laboratory for Respiratory Medicine (number SZS201617) and the Societal and Developmental Project of Suzhou (number SS201630).

\section{Disclosure}

The authors report no conflicts of interest in this work. 


\section{References}

1. Gridelli C, de Marinis F, Cappuzzo F, et al. Treatment of advanced nonsmall-cell lung cancer with epidermal growth factor receptor (EGFR) mutation or ALK gene rearrangement: results of an international expert panel meeting of the Italian association of thoracic oncology. Clin Lung Cancer. 2014;15(3):173-181.

2. Wood K, Hensing T, Malik R, Salgia R. Prognostic and predictive value in KRAS in non-small-cell lung cancer: a review. JAMA Oncol. 2016;2(6): 805-812.

3. Hicklin DJ, Ellis LM. Role of the vascular endothelial growth factor pathway in tumor growth and angiogenesis. J Clin Oncol. 2005;23(5): 1011-1027.

4. Goel HL, Mercurio AM. VEGF targets the tumour cell. Nat Rev Cancer. 2013;13(12):871-882.

5. Sandler A, Gray R, Perry MC, et al. Paclitaxel-carboplatin alone or with bevacizumab for non-small-cell lung cancer. $N$ Engl J Med. 2006; 355(24):2542-2550.

6. Garon EB, Ciuleanu TE, Arrieta O, et al. Ramucirumab plus docetaxel versus placebo plus docetaxel for second-line treatment of stage IV non-small-cell lung cancer after disease progression on platinum-based therapy (REVEL): a multicentre, double-blind, randomised phase 3 trial. Lancet. 2014;384(9944):665-673.
7. Li J, Qin S, Xu J, et al. Apatinib for chemotherapy-refractory advanced metastatic gastric cancer: results from a randomized, placebo-controlled, parallel-arm, phase II trial. J Clin Oncol. 2013;31(26):3219-3225.

8. Hu X, Zhang J, Xu B, et al. Multicenter phase II study of apatinib, a novel VEGFR inhibitor in heavily pretreated patients with metastatic triple-negative breast cancer. Int J Cancer. 2014;135(8):1961-1969.

9. Song Z, Yu X, Lou G, Shi X, Zhang Y. Salvage treatment with apatinib for advanced non-small-cell lung cancer. Onco Targets Ther. 2017;10: 1821-1825.

10. Ding L, Li QJ, You KY, Jiang ZM, Yao HR. The use of apatinib in treating non-small-cell lung cancer: case report and review of literature. Medicine (Baltimore). 2016;95(20):e3598.

11. Fang SC, Zhang HT, Zhang YM, Xie WP. Apatinib as post second-line therapy in EGFR wild-type and ALK-negative advanced lung adenocarcinoma. Onco Targets Ther. 2017;10:447-452.
OncoTargets and Therapy

\section{Publish your work in this journal}

OncoTargets and Therapy is an international, peer-reviewed, open access journal focusing on the pathological basis of all cancers, potential targets for therapy and treatment protocols employed to improve the management of cancer patients. The journal also focuses on the impact of management programs and new therapeutic agents and protocols on

\section{Dovepress}

patient perspectives such as quality of life, adherence and satisfaction. The manuscript management system is completely online and includes a very quick and fair peer-review system, which is all easy to use. Visit http://www.dovepress.com/testimonials.php to read real quotes from published authors. 